\title{
Perspective
}

PERSPECTIVE Actualité en histoire de l'art

1 | 2008

Antiquité/Moyen Âge

\section{Une question en débat : la transformation du monde romain et le rôle des barbares}

François Baratte, Jean-Michel Carrié, Walter Pohl et Gisela Ripoll

\section{OpenEdition}

1 Journals

Édition électronique

URL : http://journals.openedition.org/perspective/3489

DOI : 10.4000/perspective.3489

ISSN : 2269-7721

Éditeur

Institut national d'histoire de l'art

Édition imprimée

Date de publication : 31 mars 2008

Pagination : 19-28

ISSN : 1777-7852

\section{Référence électronique}

François Baratte, Jean-Michel Carrié, Walter Pohl et Gisela Ripoll, « Une question en débat : la

transformation du monde romain et le rôle des barbares », Perspective [En ligne], 1 | 2008, mis en ligne le 31 mars 2018, consulté le 01 octobre 2020. URL : http://journals.openedition.org/perspective/3489 ; DOI : https://doi.org/10.4000/perspective.3489 


\section{Une question en débat : la transformation du monde romain et le rôle des barbares}

François Baratte, Jean-Michel Carrié, Walter Pohl et Gisela Ripoll

Depuis I'histoire grecque, I'histoire de l'Europe a souvent été vécue comme celle d'une civilisation entourée (voire assiégée) de barbares. Pourtant, même la Grèce de Périclès n'était pas insensible à I'Orient ou à I'or des Scythes, et I'hellénisme est la marque d'une fusion entre les Grecs et "les autres».

Le sac de Rome (410) puis la chute de I'ancienne capitale de I'Empire romain (476) ont marqué les esprits rétrospectivement, aussi comme preuve inéluctable d'une décadence de l'Empire romain. Mais le récit $d^{\prime} u n e$ Rome en déclin vaincue par des peuples arrivant du fonds des steppes et franchissant le limes a été sérieusement remis en question depuis plusieurs années, tant par les découvertes archéologiques montrant l'incorporation des peuples barbares à la frontière voire au sein de l'Empire romain, que par la réflexion historique, avec le plaidoyer d'Henri-Irénée Marrou en faveur de ce qu'il souhaitait appeler l'Antiquité tardive (et non le Bas-Empire) ${ }^{1}$.

L'exposition en cours au Palazzo Grassi à Venise, Roma e i Barbari', atteste que cette histoire est toujours d'actualité, et que les problèmes d'intégrations de peuples étrangers au sein d'une civilisation ont de multiples échos de nos jours. Aussi Perspective a-t-il souhaité demander à quatre spécialistes de la période de réagir sur trois thèmes: Doit-on parler de la chute de l'Empire ou de la chute de Rome? Quel rôle les peuples dits "barbares» ont-ils joué et en quoi l'histoire et l'archéologie peuvent-ils l'éclairer? Enfin, concernant les différents langages artistiques entre la fin de l'Empire romain et le haut Moyen Âge, peut-on parler d'un art barbare?

Les avis de François Baratte (professeur à I'Université de Paris- IV-Sorbonne), Jean-Michel Carrié (directeur d'études à l'EHESS et membre du Centre Louis Gernet), Walter Pohl (professeur à l'Österreichische Akademie der Wissenschaften de Vienne) et Gisela Ripoll (maître de conférences à l'Universitat de Barcelone) autour de ces trois questions ont été réunis par un échange de courriels. [O. B.] 


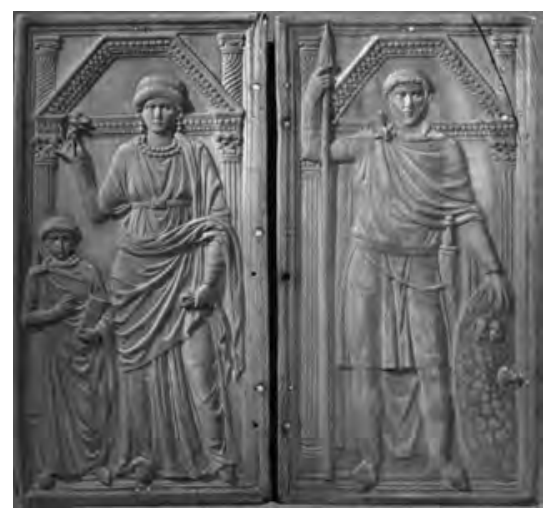

Perspective. La fin de l'Empire romain: chute de l'Empire ou chute de Rome?

Walter Pohl. Récemment, un débat "révisionniste » a éclaté : certains chercheurs, parmi lesquels John Bryan Ward-Perkins et Peter Heather, ont fortement souligné le rôle des barbares dans la chute de l'Empire romain ${ }^{3}$, et exigé un retour au vieux paradigme d'une Rome assassinée par les " barbares ». La majorité des chercheurs maintiennent, par contre, que la rupture politique et militaire n'était pas le fait essentiel, mais un procès plus fondamental de désintégration du système romain et de substitution des élites, la «transformation du monde romain ».

Une bonne partie de la polémique est inutile, parce que personne n'a maintenu qu'il y avait eu une « transition pacifique » de l'Empire aux royaumes barbares. Il a toujours été incontesté que ces transformations se

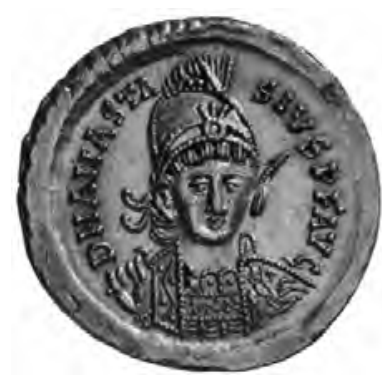

1. Diptyque de Stilicon, avec Serena, épouse de Stilicon, et Eucherius, leur enfant, vers 395, Monza, cathédrale.

2. Sou d'or d'Anastasius (491-518), frappé sous la régence de Théodoric. sont passées dans un climat de violence, avec des luttes de pouvoir et des destructions des infrastructures considérables. Mais la question fondamentale reste toujours de savoir si la violence, les faits militaires et la prise du pouvoir par les barbares ont déterminé, ou seulement accentué, les processus de transformation économique, sociale et culturelle. Des recherches récentes, par exemple dans le projet européen Transformation of the Roman World [La transformation du monde romain] ${ }^{4}$ indiquent plutôt qu'il s'agit d'un changement plus complexe et de plus longue durée, auquel beaucoup d'éléments internes ont contribué, comme la christianisation, le passage progressif des taxes publiques aux impôts fonciers privés et la délégitimisation des valeurs classiques.

Encore une observation méthodologique. Dans l'historiographie moderne, on s'est habitué à décrire "le déclin et la chute de Rome " sous forme d'oppositions binaires: Rome/barbares, Romains/Germains, chrétiens/païens, civilisation/barbares, etc. Ces modèles simplistes empêchent une compréhension approfondie, et il faut s'en débarrasser. Les « barbares » du v ${ }^{\mathrm{e}}$ ou du vi $\mathrm{i}^{\mathrm{e}}$ siècle étaient fort différents les uns des autres, cela allait des individus presque complètement romanisés (Stilicon [fig. 1], Théodoric [fig. 2]) à ceux qui appartenaient à la culture militaire romano-barbare comme Alaric ou Clovis ou aux « vrais » barbares, comme Orose appelle l'envahisseur goth Radagaisus. Tout autant, les formes de vie romaine étaient très diverses. Le déclin de ces formes de la culture des élites romaines ne signifie pas la fin complète d'une civilisation, surtout en Gaule.

Jean-Michel Carrié. La discrétion, surprenante pour un regard moderne, avec laquelle les auteurs contemporains ont pris acte de la chute de l'Empire d'Occident en 476 sous le coup des assauts barbares a été interprétée, généralement, comme une preuve du peu de modifications sensibles que cet événement aurait entraîné à leurs yeux. Les royaumes germaniques se seraient inscrits dans la continuité de la civilisation antique et des formes institutionnelles romaines qu'ils auraient davantage conservées que détruites. Toutefois, comme nous y invitait Arnaldo Momigliano voici quarante déjà ${ }^{5}$, il convient d'approfondir les raisons de ce silence assourdissant, qui peuvent être aussi inattendues que déconcertantes.

Cessons de voir dans ce silence un argument en faveur de la continuité, comme si l'établissement de royaumes barbares en partie expérimentaux à la place de la structure impériale consolidée au fil des siècles n'avait pas transformé en profondeur la physionomie de l'Europe occidentale. Pour s'en tenir à des constats objectifs, comment ne pas voir que la prise du pouvoir politique par des groupes germaniques minoritaires mais cohérents 
a déterminé une germanisation des principes mêmes de la vie en société ? Institutions civiles et militaires, catégorisation sociale à l'intérieur du groupe et typologie des rapports entre dominants et dominés tout porte la marque d'une germanisation substantielle. Comment ne pas voir que les royaumes germaniques promeuvent une société militaire appelée à dominer durablement le monde médiéval, à l'opposé des idéaux civils de l'Empire romain? Qu'ils promeuvent également, portés en cela par leur propre interprétation du christianisme, un individualisme aux antipodes des contraintes

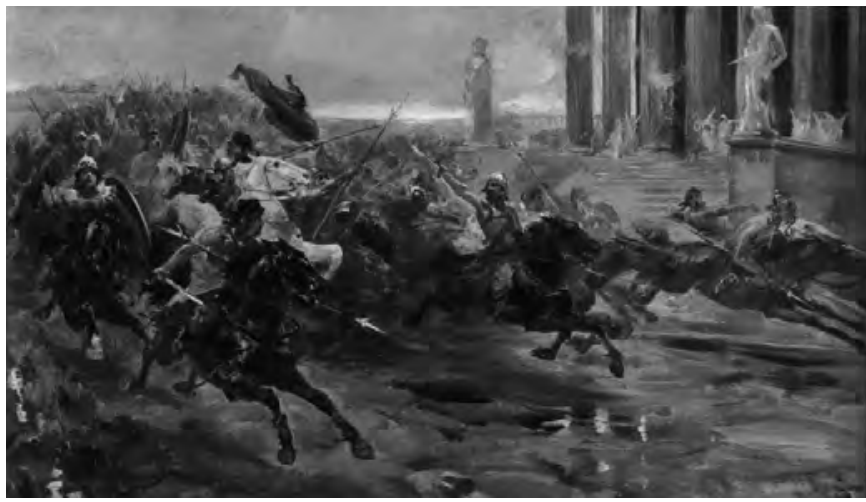
puissantes exercées sur l'homme antique par la collectivité civique. Il n'est pas jusqu'au domaine juridique où l'adoption officielle du droit romain par les nouveaux souverains n'ait visé à doter celui-ci d'une complémentarité et d'une compatibilité avec des principes fondamentalement germaniques comme le Vergeld 3. Ulpiano Checa, L'invasion des barbares, 1887, Museo Ulpiano Checa, Colmenar de Oreja (Madrid). (amendes compensatoires en cas de meurtre), le droit de la famille et de la propriété, etc. Sur cette base commune de germanisation de l'ex-Occident romanisé, l'ampleur de I'héritage institutionnel et culturel conservé varie considérablement: minimal chez les Francs et les Vandales, maximal dans les deux royaumes gothiques, sans que, dans ce cas même, la continuité assumée et revendiquée équilibre substantiellement la rupture intervenue dans le modèle social et ses assises profondes.

Dureste, ladimensioncataclysmiquedestransformationsn'apaséchappéàlaclairvoyance de certains témoins et acteurs, comme Sidoine Apollinaire (fig. 3). Deux facteurs ont, plus que tous les autres, endormi l'attention des historiens modernes sur les effets immédiats de l'installation de royaumes germaniques en lieu et place de l'Empire romain. Le premier est la permanence du latin comme langue de la communication culturelle internationale, qui a entretenu l'illusion d'une continuité factuelle, alors que cette continuité se limite à l'instrument linguistique: les mêmes termes, les mêmes signifiants latins, parce qu'ils prennent en charge de nouveaux référents, changeant alors de signifiés. Le second tient à l'apologétique chrétienne qui, cherchant une réponse aux accusations païennes contre l'usurpation d'un Dieu étranger à la tradition romaine et par conséquent incapable de protéger Rome des assauts barbares comme avaient su le faire ses divinités ancestrales, a inséré les victoires barbares dans le projet providentiel de salut universel : châtiés de leurs péchés comme l'avaient été les cités bibliques, les Romains se voyaient proposer en modèle de régénération, la supposée pureté de mœurs de leurs vainqueurs germaniques. De plus, le christianisme avait repris à son compte le schéma narratif et interprétatif des historiens classiques lisant le passé en termes de succession d'une série d'empires pour l'inscrire dans sa perspective providentialiste. Appelé à l'existence pour réaliser l'unification de l'humanité indispensable au plan de rédemption universelle, I'Empire de Rome ne pouvait être que le dernier de l'histoire du monde terrestre, dont il permettrait la fin. Bien que se plaçant dans une tout autre perspective, cette conception venait conforter le mythe de la "Rome éternelle » précédemment appuyé sur les prédictions païennes. Dans ces conditions, une « fin de Rome » paraissait inconcevable tant que n'aurait pas sonné l'heure de la fin du monde même, et la montée en puissance des barbares n'était aucunement vécue comme l'annonce d'une fin $d u$ monde imminente, ni même comme la fin d'un monde : en cela réside sans doute la déformation principale du regard porté par les modernes sur ce tournant historique. 
4. Carte des migrations du iiie au $v^{\mathrm{e}}$ siècle, d'après W. F. Volbach [L'Europe des invasions, Paris, 1967, ill. 355].
Le paradoxe veut que les barbares, nullement conscients de la fonction providentielle que leurs succès militaires leur ont value auprès des Pères de l'Église, ont fini par prendre possession à leur corps défendant, en quelque sorte, de l'appareil d'État romain qu'ils avaient jusque-là servi fidèlement en qualité de troupes dites " fédérées ». Quatre ans avant d'entrer en vainqueur dans Rome, Alaric, au nom de l'unité de l'Empire, proposait encore à Attale, l'empereur légitime, et à Honorius, l'usurpateur, de lancer ses troupes à la reconquête de I'Afrique tombée aux mains des Vandales. Le refus de cette offre de bons et loyaux services ne constitue que l'un des multiples actes suicidaires commis contre ses intérêts par l'Empire romain d'Occident, sans nul besoin d'être " assassiné de l'extérieur », pour reprendre une formulation célèbre de l'historiographie antigermanique. Rien n'a pu arrêter la déliquescence politique de l'Occident au cours du v ve siècle. L'incohérence de la situation n'a pas seulement ouvert la porte aux barbares : elle les a conduits à assumer une responsabilité politique autonome qu'ils ne concevaient même pas au départ, tant l'image impériale restait valorisée à leurs propres yeux. D'une compatibilité souple entre le titre royal à usage interne et les titres de maître des milices et patrice - les plus prestigieux dans la hiérarchie honorifique impériale -, ils sont passés à un statut de chefs d'État indépendants de fait. En apparence, les deux solutions successivement envisagées et réalisées ne se différenciaient que par la forme et la définition de l'autorité souveraine : ce qui a pu donner aux contemporains l'illusion rassurante d'une continuité. Mais dans leurs effets, ces deux régimes induisaient deux modèles profondément différents d'organisation sociale et de vie collective.

Perspective. Les peuples : ethnogenèse et identité.

Walter Pohl. La recherche traditionnelle avait marqué les migrations des peuples à travers l'Empire romain avec des flèches colorées (fig. 4). Ainsi, par exemple, on illustrait la migration des Goths de la Scandinavie aux rives de la Vistule, et d'ici aux steppes du nord de la mer Noire, à travers les provinces balkaniques, I'Italie, la Gaule méridionale et, enfin, I'Espagne. Ces idées d'une migration des « peuples » présupposent la conception d'une identité des peuples migratoires préexistante : issus d'une communauté biologique et culturelle "germanique » et demeurant jusqu'à la fin presque identiques dans leur substance.

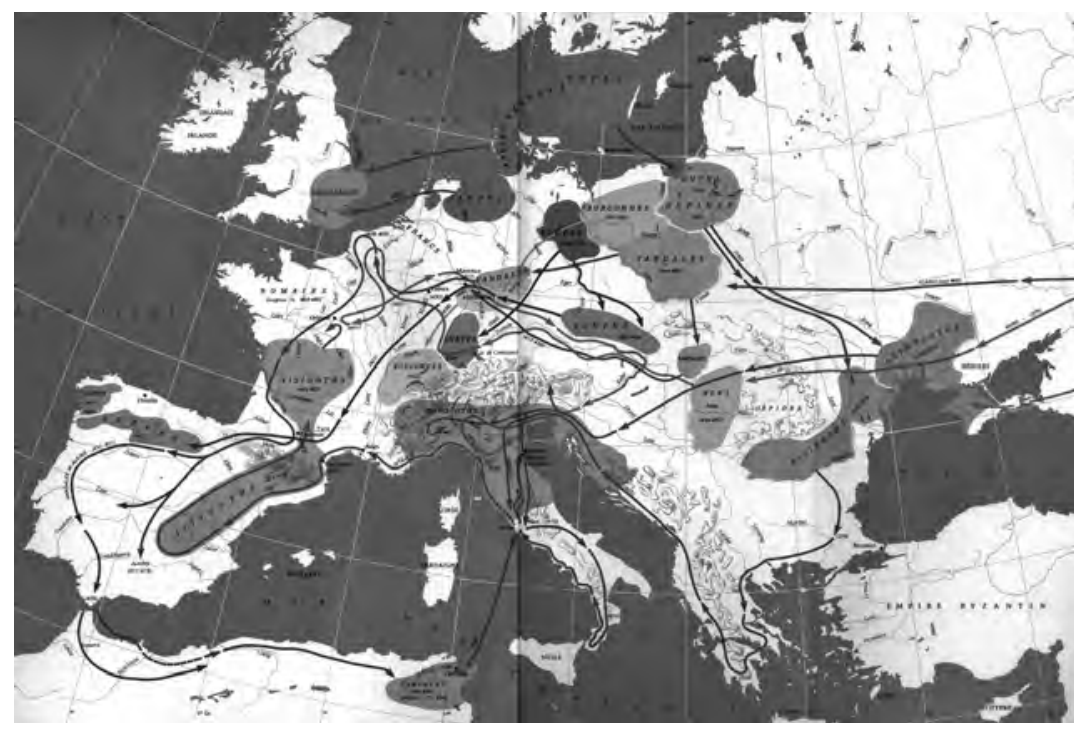

En effet, cette notion de continuité ethnique, si chère aux historiographies nationales, ne s'affirme pas dans les sources. Bien sûr, on rencontre une continuité des noms principaux à partir du

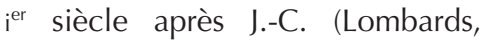
Saxons), malgré le fait que souvent leur forme exacte change (Gutones/ Gothi, Vandilii/Vandali). Mais dans les textes, il devient très clair que la composition de tous ces groupes était en transformation, surtout dans les phases de migration. Après I'attaque-choc des Huns en 375, les Goths se recomposent; les expéditions des Ostrogoths de Théodoric dans les années 473-493 

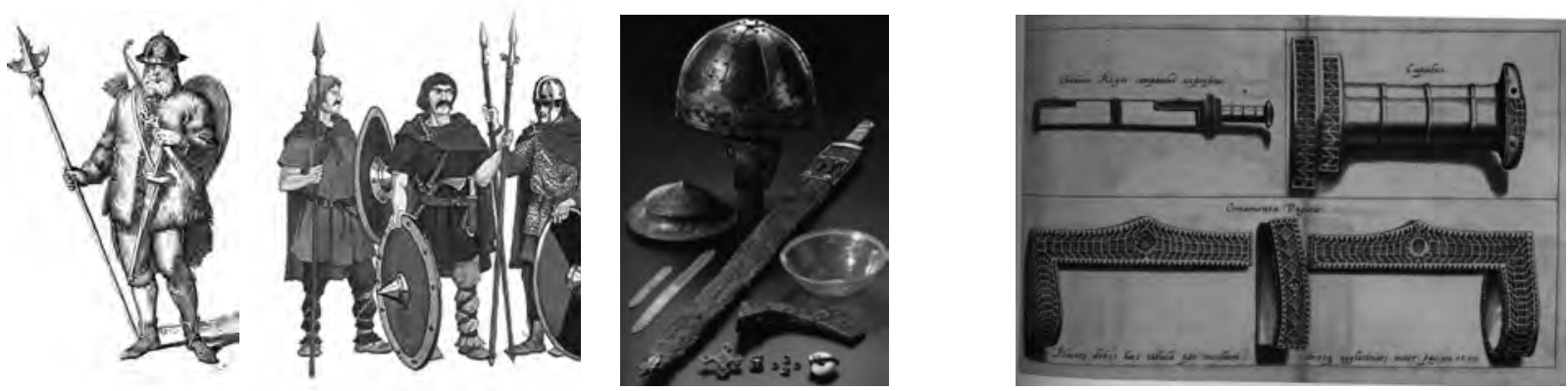

conduisent à une autre transformation fondamentale. En 568, les Lombards partent de la Pannonie pour l'invasion de l'Italie avec une armée composée aussi de Gépides, de Suèves, de Sarmates et de Bulgares (donc des peuples cavaliers de la steppe) et de Romains du Norique et de la Pannonie ; en Italie, ils se mêlent encore avec des Goths et d'autres barbares déjà sédentaires dans la péninsule, et sans doute avec des Romains. Ainsi, au cours des siècles, une minorité lombarde constitue, avec la majorité des autochtones, la population italianophone des Lombards, de même qu'en Gaule la minorité franque se mêle aux Gallo-Romains et beaucoup d'autres groupes ethniques (Alains, Alamans, Saxons, mais aussi Syriens), et que de cette manière se développe un nouveau peuple franc/français.

Pas d'ethnogenèse au début de l'histoire des peuples européens, donc, mais des transformations ethniques quasi continues pendant de nombreux siècles. Seuls les noms et les perceptions restent. Des chercheurs ont trouvé dans la cohésion de ces peuples par les mythes et les traditions communes un «mythomoteur» qui promettait un prestige extraordinaire aux individus appartenant à ces peuples guerriers (fig. 5, 6 et 7). C'est bien probable, mais il faut reconnaître que nous savons fort peu de ces mythes et normes anciens, et qu'aussi les « origines gentium » (comme s'appelle, par exemple, le mythe d'origine des Lombards) sont des stylisations latines postérieures, et non des traces authentiques de traditions primordiales. Plus fortes, bien que moins étudiées, sont les influences chrétiennes dans la stabilisation des identités ethniques dans les vieilles provinces romaines. L'Ancien Testament pouvait servir comme modèle d'un paysage ethnique lié indissolublement à I'histoire sacrée. Ainsi pouvait naître un nouveau type d'organisation du pouvoir dans l'Occident post-romain: le royaume chrétien de désignation ethnique, exprimé par exemple dans les titres royaux de forme Gratia Dei rex Francorum. Seul le christianisme pouvait, peut-être paradoxalement, garantir la légitimité et la cohésion de ces États postromains, au niveau administratif comme idéologique. Si la nation moderne est dotée de caractéristiques providentielles et quasi religieuses, cette forme de légitimation est peutêtre issue des racines distantes des royaumes ethniques après la chute de Rome.

Gisela Ripoll. L'Europe du v viècle est marquée par les grandes migrations et la création des royaumes barbares occidentaux qui ont jeté les bases de la formation de l'Europe moderne. Les diverses origines de ces peuples barbares ont laissé des manifestations artistiques, notamment vestimentaires, mises en évidence grâce aux nombreuses fouilles archéologiques, en particulier grâce à l'archéologie funéraire et à la fouille de tombes aristocratiques. Une des trouvailles les plus célèbres est la tombe du roi franc Childéric I à Tournai en Belgique, datée de la fin du ve siècle (fig. 8). Cette découverte, faite au milieu du xviie siècle, est la base même de la reconstruction de l'art barbare. À partir de cette découverte et encore aujourd'hui, les historiens de l'art caractérisent cette époque de grandes migrations par la diffusion - à partir du
5. Guerrier barbare, [Père Mariana, Historia de España, 1592].

6. Guerriers barbares, [P. Périn, E. Krähenbühl, Au cœur des royaumes barbares, "Les Francs", manuel scolaire, Nathan, 1992].

7. Trésor guerrier de Gütlingen (Bad-Würtemberg), 460-480, Stuttgart, Würtembergisches Landesmuseum.

8. Épée de Childéric, seconde moitié du v ${ }^{\mathrm{e}}$ siècle, gravure dans Jean Jacob Chiflet, Anastasis Childerici I. Francorum Regis sive Thesaurus sepulchralis Tornaci Nerviorum effossus..., Anvers, 1655. 


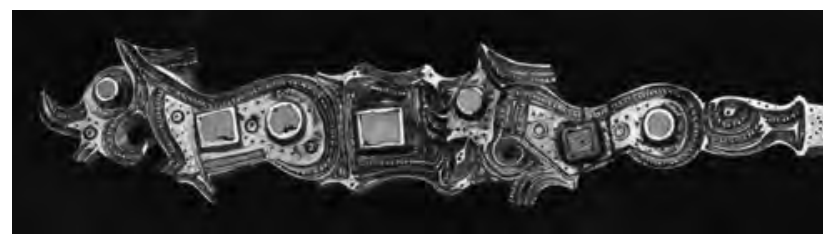

iiie siècle - d'un art dit "style polychrome » utilisé surtout pour l'orfèvrerie et les objets personnels: la profusion du décor cloisonné (géométrique ou à schématisation figurative), et/ou le décor de pierres montées en bâtes (fig. 9).

Tous les chercheurs ne s'accordent pas sur

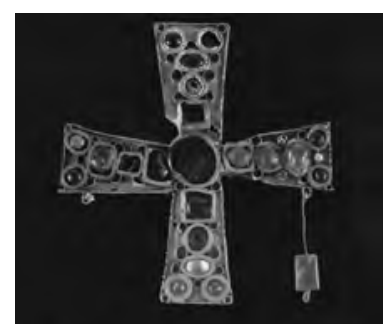

9. Épingle, v e siècle, Rouen, Musée départemental des Antiquités.

10. Croix en or du trésor de Torredonjimeno (Jaén, Andalousie), vii $^{\text {e }}$ siècle, Barcelone, Museu d'Arqueologia de Catalunya.

11. Objets découverts dans la tombe-bateau de Sutton Hoo (Suffolk) : a. assiette en argent ; b. bijoux en or.
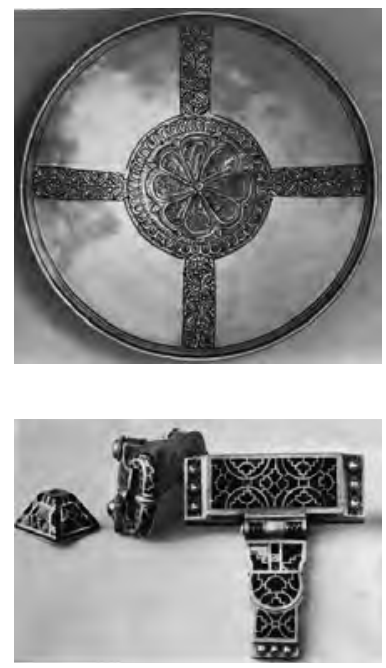

I'origine de ce style : d'aucuns estiment qu'il provient de Crimée, d'autres de Pannonie, du Kazahastan, de Géorgie, ou encore de Byzance. En fait, si l'on se fonde sur les découvertes archéologiques, le début de ce style correspond aux trouvailles faites dans les régions d'Europe centrale, avant de gagner peu à peu l'Europe occidentale, pour perdurer dans des objets, surtout liturgiques, aux viie et viiie siècles. Un bel exemple de l'importance de l'orfèvrerie et du christianisme se trouve dans la personnalité de saint Éloi (vers 588-660), orfèvre, monnayeur et évêque de Noyon (près d'Arras), à la fois conseiller de Dagobert et de Bathilde, auteur de la grande croix de Saint-Denis et du calice de Chelles (aujourd'hui perdu), ouvrages comparables aux trésors ecclésiastiques de Guarrazar (Tolède) et de Torredonjimeno (Jaén), aussi du viie siècle (fig. 10).

En coexistence avec le style polychrome, s'est développé au nord de l'Europe et en Angleterre un style ornemental dit animalier et couvrant une période qui va du v ${ }^{\mathrm{e}}$ au $x^{e}$ siècle, caractérisé par le dessin schématique d'animaux affrontés ou imbriqués. Mais réduire toutes les manifestations artistiques au «style polychrome » ou au «style animalier » semble une vision simpliste ou réductionniste du problème, car on sait par l'archéologie funéraire qu'il y avait une grande diversité de traditions vestimentaires témoignant de différences entre les multiples peuples.

Les tenants de la «nouvelle archéologie » et de la «nouvelle histoire » se refusent à voir dans la production artistique la manifestation d'une quelconque identité. Pour eux, du point de vue méthodologique, aucun objet ne permet d'identifier l'origine d'un individu ou d'un peuple. La considération de Chris Wickham, à propos des objets de parure personnelle trouvés en Italie lombarde, est bien connue : "Un homme ou une femme avec une broche de style lombard n'est pas plus nécessairement un Lombard qu'une famille de Bradford avec une Toyota n'est japonaise ; les artefacts ne sont pas un signe d'ethnicité fiable $»^{6}$. Néanmoins, I'art de ces peuples, dotés d'une forte personnalité, n'est rien d'autre que le fruit de la culture romaine et byzantine en interaction avec leur propre tradition artistique, surtout vestimentaire : la formation culturelle des rois et de l'aristocratie barbare, en effet, plongeait ses racines dans la civilisation romaine, tant occidentale qu'orientale, depuis l'existence même de l'Empire et ses contacts au-delà des frontières. À nouveau, la tombe du roi franc Childéric I (vers 437vers 481), montre cette dualité : le roi est enseveli comme un chef barbare, avec ses chevaux, ses armes et ses bijoux, et comme une autorité romaine, revêtu d'un manteau de pourpre et de ses insignes (comme par exemple une monnaie de l'empereur Léon irr). De même, la tombe-bateau princière anglo-saxonne fouillée en 1939 à Sutton-Hoo (Woodbridge, Suffolk) montre un individu enseveli avec des objets saxons, mais aussi avec des objets provenant du continent, notamment des objets d'origine franque et méditerranéenne (fig. 11).

\section{Perspective. $u n$ art barbare ? les différents langages artistiques entre la fin de l'Empire romain et le haut Moyen Âge}

Gisela Ripoll. Les «siècles obscurs »- the "dark ages »: c'est ainsi que les historiens du $x i x{ }^{e}$ siècle et de la première moitié $d u x^{e}$ siècle qualifient l'époque qui va de l'Empire romain au Haut Moyen Âge, c'est-à-dire grosso modo du ive au $x^{\mathrm{e}}$ siècle. Les travaux historiques, archéologiques et linguistiques réalisés par la suite ont montré la spécificité 
et la grande richesse de cette période, baptisée dès lors Antiquité tardive $\mathrm{p}^{7}$ Depuis le début $\mathrm{du} \mathrm{xx}^{\mathrm{e}}$ siècle et les recherches pionnières d'Alois Riegl ${ }^{8}$, l'art ou les arts de l'Antiquité tardive ne sont plus considérées comme décadents mais, au contraire, à égalité avec les manifestations artistiques de l'âge classique.

Comme l'a écrit Henri-Irénée Marrou: "Il faudrait enfin consentir à admettre que l'Antiquité tardive n'est pas seulement l'ultime phase d'un développement continu ; c'est une autre Antiquité, une autre civilisation, qu'il faut apprendre à reconnaître dans son originalité et à juger pour elle-même et non à travers les canons des âges antérieurs ${ }^{9}$.

Cette époque est marquée par deux élémentsclés - l'enracinement du christianisme et l'apparition des barbares -, et par un siècle charnière, le viii ${ }^{\text {e }}$, durant lequel se cristallisent des spécificités régionales.

Il suffit de parcourir rapidement les salles ou le catalogue de l'exposition Rome et les Barbares qui se tient au Palazzo Grassi, à Venise ${ }^{10}$, pour voir que, de la fin de l'Empire romain au Haut Moyen Âge, l'évolution du langage artistique est placée sous le signe de la continuité avec, bien entendu, des spécificités liées aux ateliers, aux artistes, aux commanditaires et,

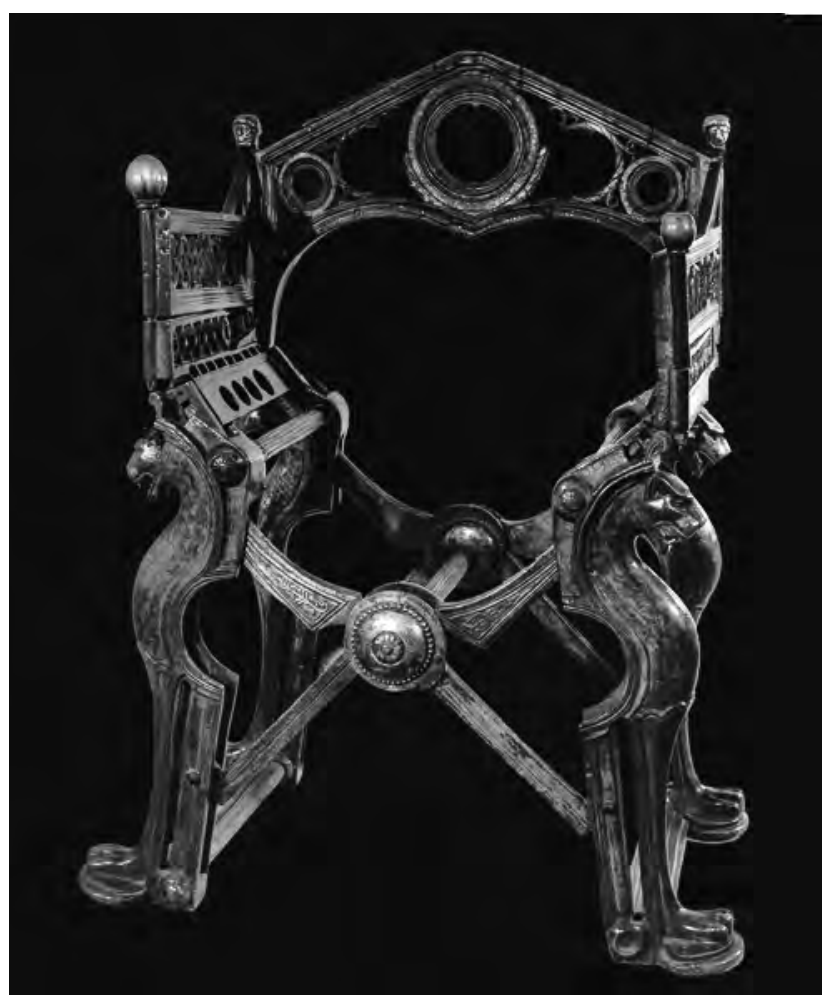
naturellement, aux zones géographiques ${ }^{11}$. Un bon exemple de cette continuité du monde romain se trouve dans le " trône de Dagobert » (Cabinet des Médailles, BnF, Paris ; fig. 12) daté du vii e siècle, à mi-chemin entre la sella curulis romaine et le trône médiéval.

Le développement artistique de l'Orient, qu'il s'agisse de l'Empire byzantin ou de l'art islamique, est très différent de celui de l'Occident, où se sont créés des royaumes barbares marqués par une profonde empreinte romaine - les Anglo-Saxons en Angleterre, les Francs mérovingiens en Gaule, les Ostrogoths en Italie, les Wisigoths dans la péninsule ibérique. La puissance de ces sociétés respose essentiellement sur les cavaliers et les guerriers, ce qui nécessite une maîtrise de la métallurgie et de l'orfèvrerie exceptionnelles. La personnalité des différentes cultures de ces royaumes est marquée par la production d'objets à la mode - tout spécialement fibules, plaques-boucles (fig. 13). Malgré la diversité des productions artistiques de chacun de ces royaumes, tous portent déjà en germe les bases de la renaissance artistique carolingienne. L'unicité de ces langages vient surtout du fait du christianisme.

François Baratte. On rappellera en effet, du point de vue historiographique, l'importance qu'ont eue, à la fin du xix e siècle, les travaux de l'école de Vienne, et notamment ceux

12. Siège dit " trône de Dagobert ", vii ${ }^{\mathrm{e}}$ ou viiie siècle (?), dossier et accoudoirs, $2^{\mathrm{e}}$ moitié du ix ${ }^{\mathrm{e}}$ siècle, Paris, Bibiothèque nationale de France, Département des monnaies, médailles et antiques.
13. Plaque-boucle de ceinture de la tombe de la reine Aregonde, fin du $\mathrm{vi}^{\mathrm{e}}$ siècle, Saint-Germain-en-Laye, Musée des Antiquités nationales. d'Alois Riegl, dans le changement d'attitude des historiens de l'art à l'égard de l'art romain, et en particulier l'art romain tardif. L'attention s'est alors focalisée sur un domaine particulier, longtemps laissé à l'arrière-plan, celui des "arts mineurs", le terme est significatif. L'un des grands mérites de Riegl est

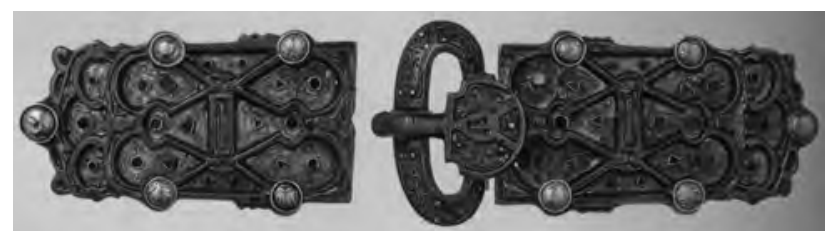




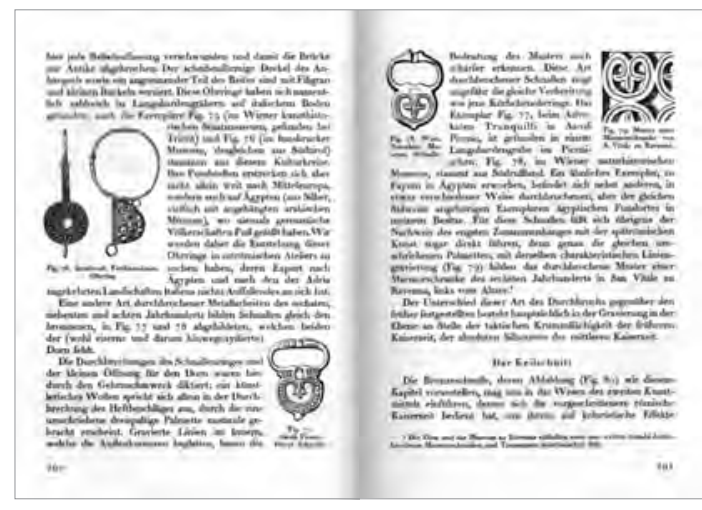

d'avoir précisément montré que certains de ces objets, parfois modestes, étaient révélateurs d'une esthétique nouvelle - celle de l'Antiquité tardive (fig. 14). Riegl plaçait ainsi au premier plan non plus l'art officiel de l'Urbs, mais l'art « provincial », et au-delà, l'art « barbare ». Mais il avait très clairement pressenti que la transformation s'ancrait effectivement dans l'art du monde romain.

Il me paraît tout à fait intéressant d'observer sa démarche en regard de celle d'un analyste aussi profond de l'art romain que l'a été Ranuccio Bianchi Bandinelli, qui avait compris lui aussi en profondeur le caractère fondamental de l'évolution, le passage, pour reprendre ses termes, de "Rome, centre du pouvoir » à " la fin de l'art antique ", sans la définir dans ses

14. Alois Riegl, Spätrömische Kunstindustrie (...), Vienne, 1901-1927, p. 291-292.
15. Obélisque de Théodose, vers 390, détail du relief ouest de la base : la soumission des barbares, Constantinople. détails, parce qu'il s'en était tenu, délibérément, à certaines formes d'art, la sculpture pour l'essentiel, qui n'est peut-être pas la plus représentative de l'Antiquité tardive ${ }^{12}$.

Quant aux royaumes barbares, il convient de souligner, comme le fait Gisela Ripoll I'empreinte de Rome sur eux, ce que montre l'exposition de Venise, Roma e i Barbari, quelque décevante qu'elle soit sur tous les plans. Ainsi des Vandales en Afrique : les textes comme l'archéologie montrent bien ce souci, pendant un siècle, de 429 à 533, de se couler dans le moule romain : c'est de cette façon que l'on peut comprendre la description que fait le poète Luxorius, dans I'Anthologie latine, du palais des rois vandales près de Carthage, ou d'un superbe groupe thermal. Sans cela, la « renaissance carolingienne » ne s'expliquerait pas.

Il convient aussi sans doute de tenir compte d'une question de langage. Parler d'un « art barbare » signifie au fond qu'il y a en face un « art romain » senti comme tel par les contemporains. Certes, on n'oubliera pas qu'il y a de la part des Romains un réel sentiment de différence avec les peuples venus de l'extérieur : qu'on pense aux nombreuses allusions que fait Sidoine Apollinaire dans ses poèmes et dans ses lettres, autour de 460-480, aux barbares, aux Burgondes et aux Wisigoths notamment, qu'il avait côtoyés aussi bien à Lyon qu'en Auvergne, quand il vivait " au milieu de hordes chevelues», obligé de supporter "les chansons du Burgonde gavé qui s'enduit les cheveux de beurre rance » (Poème XII, v. $3-7)^{13}$. À la toute fin du ive siècle, les reliefs de la base de l'obélisque de Théodose, à Constantinople, montrent les peuples barbares, vêtus de fourrure ou en costume oriental (fig. 15). Mais cela n'implique pas l'existence effective, surtout dans le domaine concerné au premier chef par « l'art barbare », celui de l'équipement personnel, d'objets reconnus comme identitaires.

Il est sans doute bon de replacer dans cette perspective notre appréciation du rôle des barbares qui, de toute évidence, marquent la période sur le plan historique.

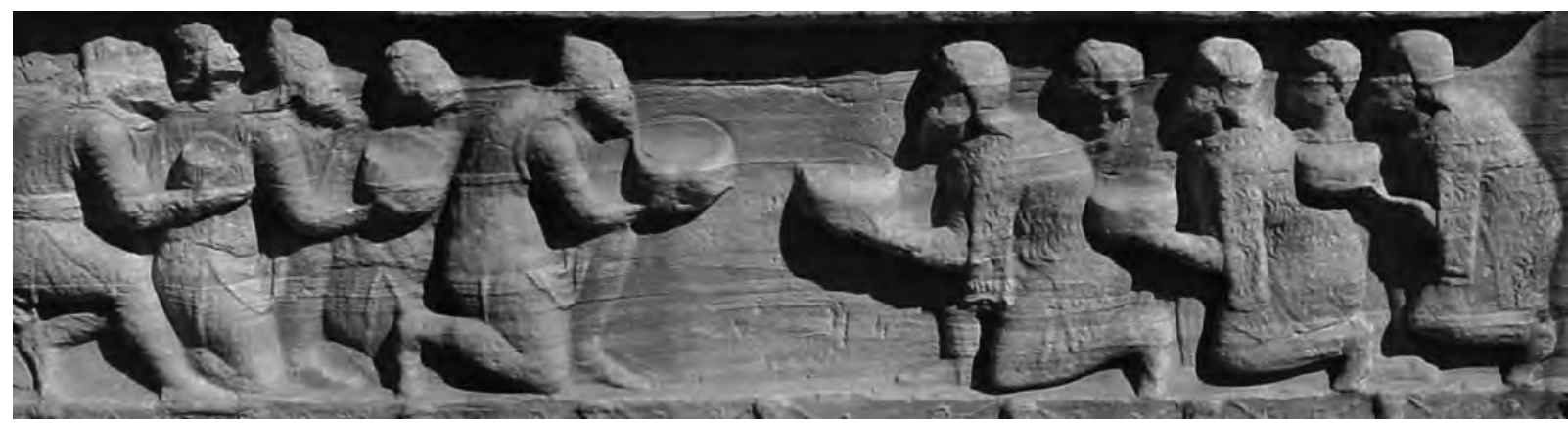


Encore le problème mériterait-il, sur le plan de l'art, une réflexion globale, et pas seulement "occidentale». II conviendrait de voir précisément, et simultanément, ce qui se passe dans l'est de la Méditerranée, à Constantinople, mais pas exclusivement: une part importante de l'évolution de l'art y plonge aussi ses racines, même si le développement artistique y est effectivement très différent. II convient de pas négliger non plus ce qui se

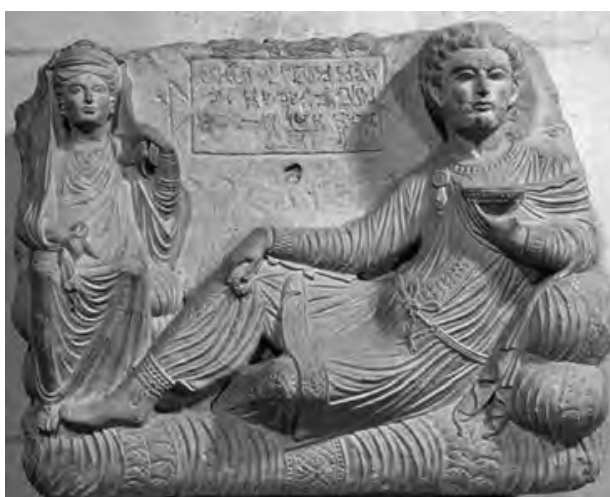
passe à l'Est de la Méditerranée dans la naissance et la diffusion du "style polychrome ». Les objets conservés ne sont pas si nombreux, mais les exemplaires conservés et leur représentation - par exemple les sculptures funéraires de Palmyre (fig. 16) - témoignent du goût pour les bijoux qui mêlent I'or et les pierres. On doit souligner aussi la présence à Rome, dans l'art impérial, dès la fin du iiie siècle, d'un goût analogue : sur le fourreau de la spatha des tétrarques de Venise, par exemple, sans parler des bijoux que l'on voit portés par les femmes sur les mosaïques de Piazza Armerina, un peu plus tard dans le courant du iv e siècle (fig. 17).

C'est pourquoi on ne peut qu'adhérer tout à fait à l'idée suivant laquelle l'art des peuples «barbares » est le fruit à la fois de leur personnalité et de la culture romaine et byzantine.

Jean-Michel Carrié. Par-delà ces progrès dans la connaissance des échanges entre culture romaine et cultures barbares, une appréciation proprement historique de la portée de la " chute de Rome » exige qu'on en finisse une fois pour toutes avec la valorisation idéologique de deux "civilisations ", la romanité et la germanicité, concepts qui ne sont guère plus que les appendices culturels des antagonismes géopolitiques entre nations européennes au cours des cinq derniers siècles $\left(\mathrm{xvi}^{\mathrm{e}}-\mathrm{xx} \mathrm{x}^{\mathrm{e}}\right)$. Nous n'en sommes plus, en 2008, à donner crédit au mythe historiographique, introduit à l'époque de la Renaissance et amplifié par l'âge des Lumières, $d^{\prime}$ une destruction des formes supérieures de la civilisation antique sous les coups des Goths. Le passage du monde antique au monde médiéval n'est pas à prendre comme un « mieux » ou comme un " pire ».

Comment, par contre, ne pas s'interroger sur les raisons pour lesquelles l'Europe du xiie siècle présente un aspect si différent tant de l'Occident romain du v ve siècle que de I'Empire byzantin contemporain qui avait, lui aussi, évolué en sept siècles, mais en conservant les cadres généraux de l'héritage romain et du premier christianisme oriental ? II ne s'agit pas de sous-évaluer l'importance du legs antique dans la civilisation européenne moderne. Mais à voir les blocages de divers types (économiques ou culturels) auxquels se heurtait la civilisation antique au terme de son parcours, à voir comment ces blocages ont largement persisté dans l'Empire byzantin conservant dans son évolution même les cadres généraux de I'héritage romain, au point qu'il soit passé, finalement, sous la coupe d'un Occident reparti sur de nouvelles bases, celles en particulier d'une rationalité économique qui n'a cessé ensuite de singulariser l'aventure européenne par rapport au reste du monde, on est en droit de se demander si l'Empire romain, après avoir porté à leur dernier stade de réalisation les potentialités de la civilisation antique, n'en avait pas atteint les limites indépassables. Sans pour autant créditer les barbares d'avoir été porteurs d'un modèle alternatif conscient et organisé, ceux-ci n'auraient-ils pas été les déclencheurs aveugles et en partie accidentels

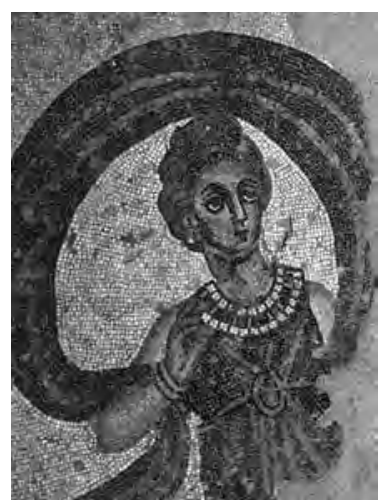

16. Relief funéraire, $1^{\text {re }}$ moitié du iii $^{e}$ siècle, Palmyre (ancienne Tadmor, Syrie), Paris, Musée du Louvre.

17. Mosaïque de Piazza Armerina, Sicile, iv ${ }^{\mathrm{e}}$ siècle (?). 
d'un nouveau cours de l'histoire, discernable non pas à son point de départ, mais à son point d'arrivée ? N'ont-ils pas été, bien inconsciemment - voire, à l'inverse de leurs propres aspirations -, les instruments d'une rupture historique à long terme indispensable pour que I'histoire cessât de tourner en rond ou de faire du surplace ? Or, un raisonnement analogue ne pourrait-il pas être tenu dans le domaine artistique? Les barbares n'ont pas davantage interrompu temporairement un âge d'or qui aurait mis dix siècles à revenir.

La Renaissance européenne n'est pas la restauration de l'Antiquité et ceux de ses courants qui se limitaient à un tel programme ont été les moins créateurs (pour ne prendre qu'un exemple, les théoriciens de l'aristotélisme littéraire appliqué au théâtre). Livré à ses propres potentialités et perpétuant ses blocages, le monde antique n'aurait pas davantage produit la peinture du cinquecento que la Compagnie des Indes ou la Banque d'Amsterdam. Assurément, ces innovations historiques n'étaient pas davantage à la portée des barbares du $v^{e}$ siècle, mais leurs conditions d'apparition se seraient-elles réalisées si ces mêmes barbares n'avaient pas mis un terme au monde antique? Une telle interrogation relèvet-elle, aujourd'hui encore, du blasphème culturel ?

1. Henri-Irénée Marrou, Décadence romaine ou Antiquité tarddive : iii ${ }^{\mathrm{e}}{ }^{-i v^{e}}$ siècles, Paris, 1977.

2. Roma e i Barbari, Jean-Jacques Aillagon, Yann Rivière, Roberto Umberto éd., (cat. expo., Venise, Palazzo Grassi, 26 janvier - 20 juillet 2008/Bonn, Kunst- und Ausstellungshalle der Bundesrepublik Deutschland, 22 août 2008 - 7 décembre 2008) ; trad. fr. : Rome et les Barbares, Paris/Dijon, 2008.

3. John Bryan Ward-Perkins, The fall of Rome and the end of civilization, Oxford, 2005 ; Peter Heather, The fall of the Roman Empire, Londres, 2005.

4. Ce programme de recherche, mené de 1993 à 1998 par la European Science Foundation, a donné lieu à des publications dans la série Transformation of the Roman World, Leyde/Cologne/New York, 1997-2004.

5. Arnaldo Momigliano, Alien Wisdom : The Limits of Hellenization (1976); trad. fr. : Sagesses barbares, Paris, 1979.

6. " a man or a woman with a Lombard-style brooch is no more necessary a Lombard than a family in Bradford with a Toyota is Japanese; artefacts are no secure guide to ethnicity ", Chris Wickham, Early Medieval Italy : Central Power and Local Society 4001000, Londres/Totowa, 1981, p. 68.

7. Arnold Hugh Martin Jones, The Later Roman Empire, 284-602. A social economic and administrative survey, Oxford, 1964 ; Peter Brown, The World of
Late Antiquity from Marcus Aurelius to Muhammad, Londres, 1971 ; Averil Cameron, The Mediterranean World in Late Antiquity, AD 395-600, Londres/New York, 1993 ; Glen Warren Bowersock, Peter Brown, Oleg Grabar éd., Late Antiquity. A Guide to the Postclassical World, Cambridge (Mass)/Londres, 1999.

8. Alois Riegl, Stilfragen. Grundlegungen zu einer Geschichte der Ornamentik, Berlin, 1893 ; trad. fr. : Questions de style. Fondements d'une histoire de I'ornementation, Paris, 2002 ; Alois Riegl, Die Spätrömische Kunst-Industrie nach den funden in Osterreich-Ungarn, Vienne, 1901, 2 vol. ; trad. angl : Late Roman art industry, Rome, 1985. Voir Sandro Scarrocchia, Studi su Alois Riegl, Bologne, 1986.

9. Voir Marrou, 1977, cité n.1, p. 13.

10. Voir Roma e i Barbari, 2008, cité n. 2.

11. Voir par exemple, en dernier lieu, Roland Recht, Le grand atelier. Chemins de l'art en Europe, $v^{\mathrm{e}}$-xviii ${ }^{\mathrm{e}}$ siècle, Bruxelles, 2008.

12. Ranuccio Bianchi Bandinelli, Roma : I'arte romana nel centro del potere, Rome, 1969 ; trad. fr. : Rome le Centre du pouvoir, Paris, 1969. Ranuccio Bianchi Bandinelli, Roma: La fine dell'arte antica, Rome, 1970 (2002) ; trad. fr. : La fin de l'art antique : l'art de l'Empire romain de Septime Sévère à Théodose $i^{e r}$, Paris, 1970.

13. Sidoine Apollinaire, Poèmes, trad. A. Loyen, Paris, 1960. 